\section{Visualisation of Native Surfaces by Two-Step Molding}

\author{
Stanislav N. Gorb
}

Max Planck Institute for Metals Research, Stuttgart, Germany, s.gorb@mf.mpg.de

\section{Introduction}

One of the most important tasks of microscopy is to provide information about structures in their natural state. Since most life science microscopy procedures require fixation, dehydration, drying, and sectioning, diverse artefacts are unavoidable. It is also possible to dehydrate the water content of a specimen by freeze-drying. However, even relatively stable surface structures are changed during such treatments. Methods of liquid substitution and freezingsubstitution show good results for biological specimens with a waxy solid coverage (Ensikat and Barthlott, 1993) and soft specimens in a mechanically deformed state (Gorb et al., 2000).

However, all these methods have certain restrictions for some types of specimens. For example, there are numerous biological surfaces covered with secretory fluids. Some specimens bear solid waxy coverings, which can be partly dissolved and washed out in organic solvents, such as ethanol, acetone, or propylenoxide. Often one would like to visualise dynamic processes (growth, deformation, or crystallisation) under environmental conditions, but at the high resolution in the SEM. An even more challenging specimen

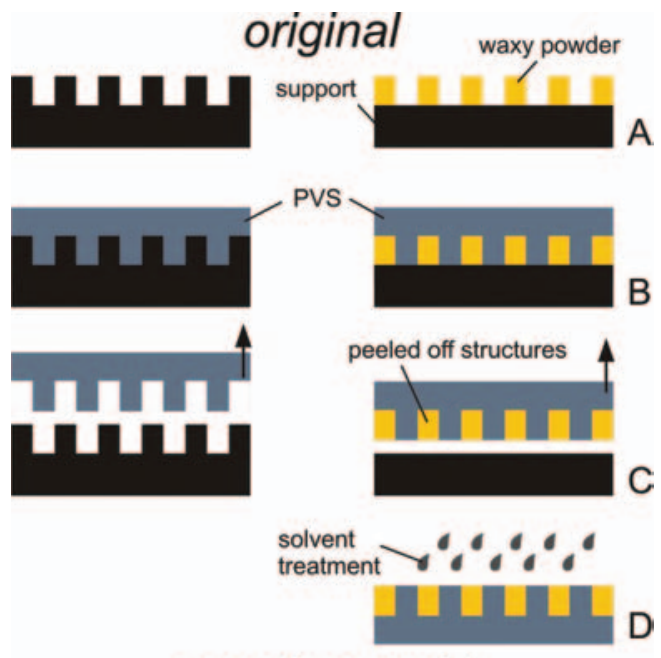
preparation situation is when your object of interest A has dimensions in the range of square meters, and there is no possibility to cut it into pieces. Finally, one can wish to visualize hundreds of natural surfaces from a field trip to a rain forest or to a desert. How can we combine such challenging requirements with

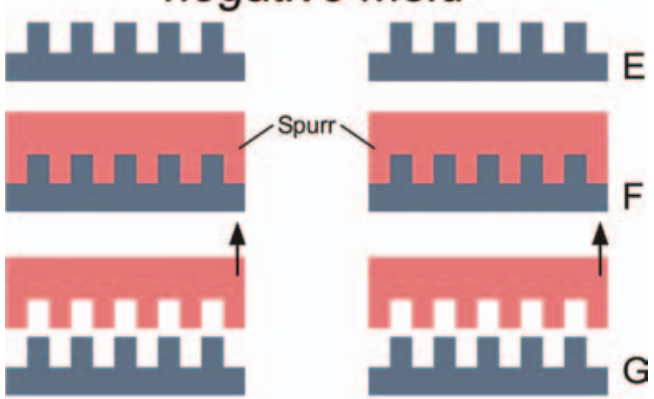

positive mold
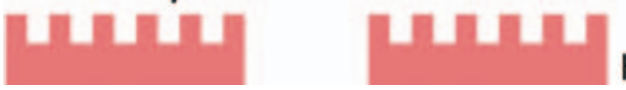

Figure 1. Diagram of the method described. Left column: two step molding technique. Right column: lost wax molding technique for brittle surface microstructures soluble in organic solvents. problems. We have used this method for the last eight years for various tasks ranging from applications in zoology, botany, and biomechanics to tribology and materials science. In this article we present applications of the method for challenging samples such as high-aspect-ratio microstructures, fragile wax crystals, and fluid coverage. This method is completely different from the replication technique used in some TEM procedures (Schwarz and Gorb, 2003), which implies carbon-platinum coating of the specimen with further removal of the thin C-Pt film from the specimen by $\mathrm{HF}$ acid. We also report on typical problems and restrictions for the use of the method.

\section{Method description}

The dental wax cast technique (Fig. 1) is a simple SEM method that makes repeated observations of the same native surface possible. For this purpose, we use a two-component dental wax (President light body, Coltene, Switzerland) to obtain negative casts from a living surface at room temperature. This material contains silicone polymer polyvinysiloxane (PVS) as its main component. There have been many different brands of silicones tested in our lab; however, the above material showed the best performance in almost all types of applications, presumably due to its low viscosity in the fluid state. Both components are mixed together in the proportion 1:1, quickly (10-20 s) stirred with a stick and immediately applied and spread onto the surface of interest. The most important requirement for the surface of interest is its immobility during polymerisation time. After polymerisation (5-7 $\mathrm{min}$ ), the silicone negative mold can be easily peeled off the surface. The mold can be further filled out by Spurr's low-viscosity resin (Spurr, 1969) and polymerized overnight at $70^{\circ} \mathrm{C}$. The hardened positive resin molds has to be removed from the silicone negative mold, mounted on an SEM holder, coated with gold-palladium and then examined in an
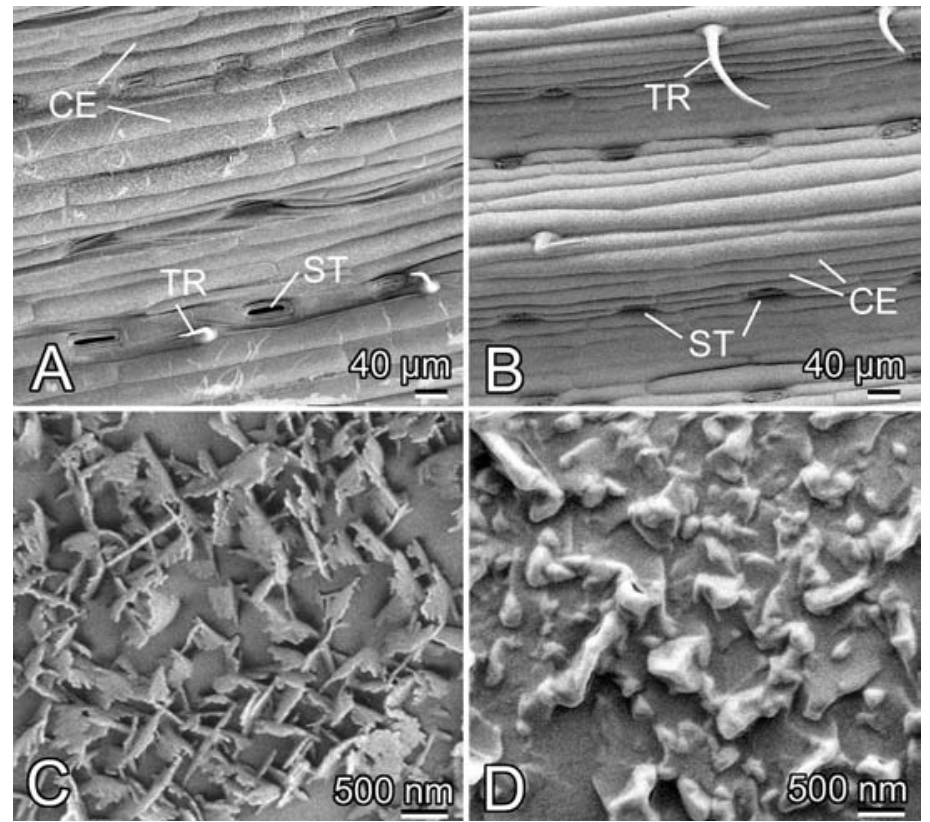

Figure 2. Leaf surface of the plant Triticum aestivum. A, C. Originals (Cryo-SEM, Hitachi S-4800 equipped with the Gatan ALTO-2500 cryo preparation system). $B, D$. Molds prepared according to the lost wax molding technique. A, B. Low magnification micrographs demonstrating molding quality of such microstructures as cells, micropores, and trichomes. $C, D$. High magnification images showing molding quality of fragile nanostructures, such as crystalline waxes. CE, cells; ST, stomata; TR, trichomes. 
SEM. This method demonstrated good results with relatively long microstructures $(10-30 \mu \mathrm{m})$ with aspect ratios of 1-20, as well as with small structures (down to $0.02 \mu \mathrm{m}$ ).

In some special applications, when the surface is covered with small brittle wax crystals (e.g. some surfaces in plants and insects), an additional step has to be applied (Fig. 1). Since the wax crystals adhere more readily to the fluid silicone mold material than to the substrate of interest, they are peeled off by the silicone and removed from the original surface. The positive mold prepared according to the method described above usually does not replicate the fine structure of the crystals. For this case, the peeled off negative mold should be washed in chloroform at room temperature for 2-3 min, in order to dissolve and remove the wax crystals attached to the mold. After a short air drying procedure $(10 \mathrm{~min})$, the negative molds can be used for preparation of positive molds (Koch et al., 2007). This technique demonstrates excellent replication ability for microstructures, such as cells, micropores, trichomes (Fig. 2 A, B) and even shows the presence of micro- and nanostructures, which would be otherwise dissolved in organic solvents (Fig. 2 C, D). The quality of the molding of these small structures with a high aspect ratio remains rather low, because the size of the crystals is already within the range of dimensions where the molding technique is limited presumably due to the viscosity of the dental wax and its wettability on nanoscopically rough surfaces.

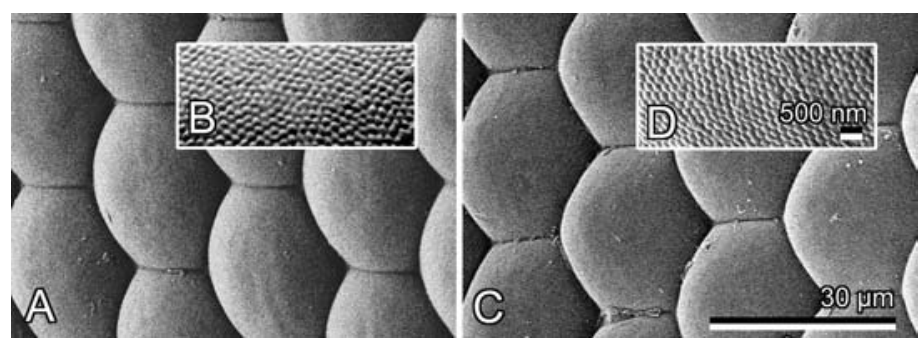

Figure 3. Ommatidia surface of the eyes of the sphindid moth Amorpha populi L. (Lepidoptera, Sphindidae). A, B. Original. C, D. Mold.

\section{Visualisation of small surface structures}

This method has shown very good results in visualisations of solid surfaces with a resolution better than $20 \mathrm{~nm}$. This resolution was obtained in the study of nanostructured surfaces obtained from metal vapour deposition. However, this high resolution is possible only for convex surface features with an aspect ratio under 1. For example, biological nanostructures with this aspect ratio, such as the ommatidia grating of insect eyes (nanostructures with the diameter of 100-200 nm), were almost perfectly molded (Fig. 3).
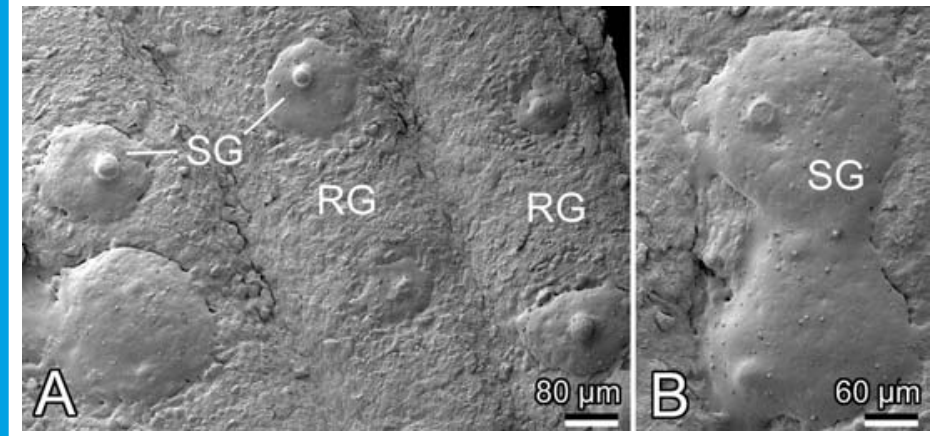

Figure 4. Molds of sweat on a human finger. Note islands of the fluid (SG) coming out of the pores of sweat glands. RG, ridges of the finger skin.

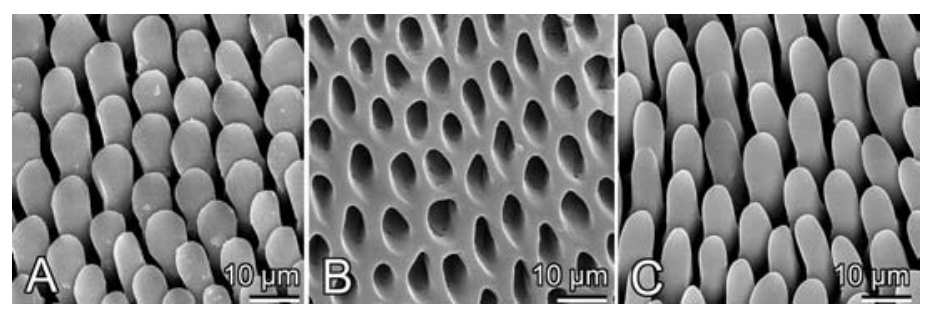

Figure 5. Fields of microtrichia of the damselfly Pyrrhosoma nymphula (Zygoptera, Coenagrionidae) head arresting system (for details see Gorb, 1999b). A. Microtrichia of the locking field on the rear surface of the head. B. Negative mold of the pattern of microstructures. C. Positive mold of the same surface.

\section{Visualisation of fluids}

In a study of the interlocking covering system of beetle wings, we compared samples of the same surfaces obtained with different preparation techniques. The results have clearly showed that native surfaces of these areas of insect cuticle are covered with a liquid secretion (Gorb, 1999a). Fluid sweat can be visualised on human fingers (Fig. 4). This example shows that this approach may be very useful in the visualisation of surfaces (with or without fluid) that normally otherwise cannot be studied in a native condition in the SEM. Human skin and teeth, as well as the surfaces of other organisms, non-living surfaces of large dimensions, where cutting of the samples is impossible (specimens of animals and plants, valuable pieces of art) are a few examples, where the use of this technique is desirable.

\section{Molding of structures with high aspect ratio and hierarchi- cal structures}

The method was applied for molding long cuticle outgrowths in some insect species. We have previously reported on the molding of the complex pattern of the high-aspect ratio microstructures of
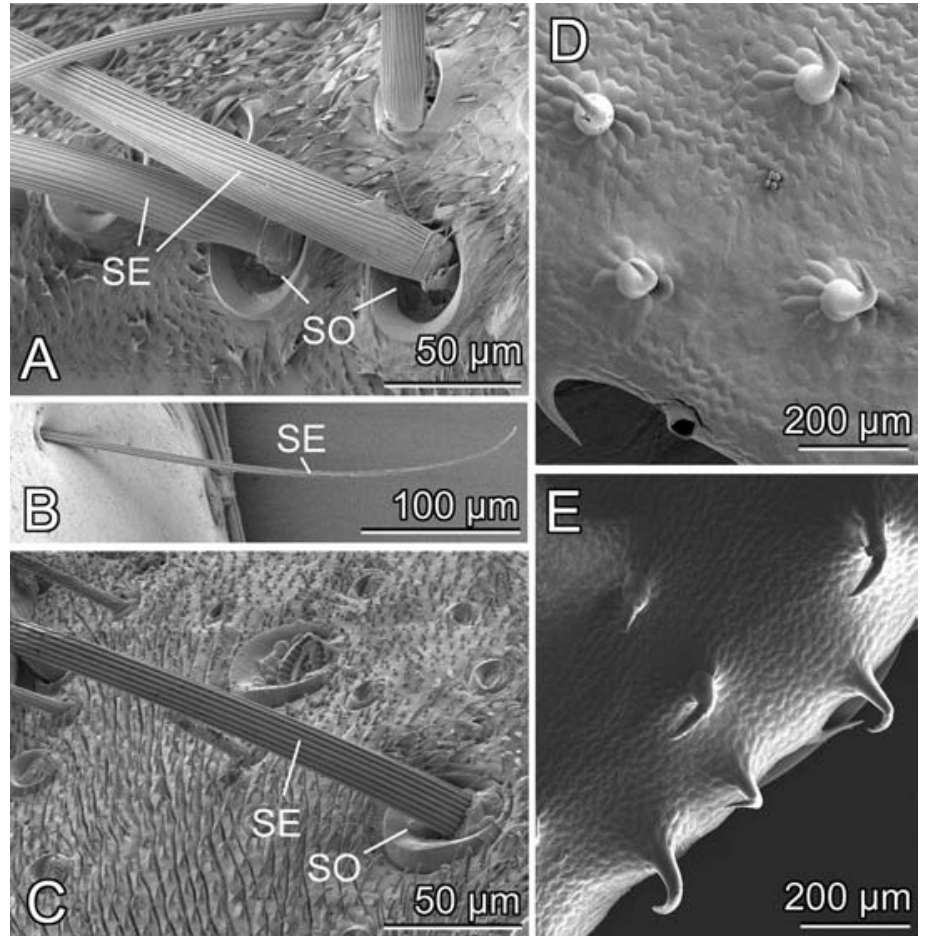

Figure 6. Results of molding of structures with a high aspect ratio $(A-C)$ and with intersections (D-E). A-C. Head setae of the fly Calliphora vicina (Diptera, Calliphoridae). D-E. Leaf hooks of the plant Galium aparine. A, D. Originals. B, C, E. Molds. SE, setae; SO, socket. 


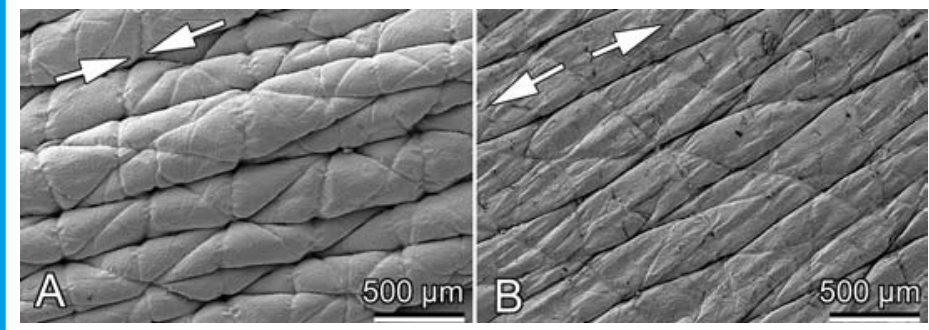

Figure 7. Use of the molding technique for visualisation of dynamic processes on the surfaces, which otherwise cannot be studied in the SEM. Pattern of the human wrist skin under compression (A) and under tension (B). Arrows show directions of compression and tension.

the dragonfly arresting system (Scherge and Gorb, 2001) (Fig. 5). Setae of flies Calliphora vicina, with an aspect ratio of about 30, were the tallest structures, we successfully molded (Fig. 6 A-C). Even structural details of the hair surface, such as grooves running longitudinally along the hair, are perfectly replicated (Fig. 6 C).

Normally, it is difficult to mold surface outgrowths having intersections. It is even more difficult to mold them twice. However, some mechanically stable macrostructures with intersections, such as barbs of the plant Galium aparine (Gorb and Gorb, 2002), can be successfully molded (Fig. 6 C, D). Good results were obtained also for etched metal surfaces with strong hierarchical surface organisation from the micro- down to the nanoscale (Gorb, personal communication).

\section{Visualisation of dynamic processes}

Two step molding technique opens a great possibility to observe changes of the same surface during growth. The sequences of the momentary snapshots of the changing surface can be obtained during growth or deformations under external load. We have visualised the surface of human skin deformations under applied shear force (Fig. 7). This method can be used in combination with force measurements and is especially interesting for applications in surface science and tribology to observe morphological changes of the surface under influence of friction and adhesion. For example, the method was successfully applied in studies of the wear dynamics at the micro- and nanoscale in car engines (Scherge and Gorb, personal communication).

\section{Other potential fields of applications}

In addition to the applications described above, the method is especially interesting for use in the field. Instead of carrying a large amount of fixatives, the desired specimen pieces can be simply molded directly in the field and taken in the form of PVS negatives back to the lab for preparation of positive molds and further SEM observations. The negatives can be kept for a long time and a number of positive molds can be prepared, which is interesting for multiplication and distribution of the samples of particular interest. In some cases, SEM investigation of valuable holotype specimens from museum collections is required. In order to prevent damage of the material during transportation and preparation procedure, molds can be made and distributed instead of original specimens.

\section{Limitations}

The method described here has been successfully applied for visualisation of fluids on various surfaces, nanostructures with low aspect ratio, and microstructures with high aspect ratio (Fig. 8). However, there are some limitations of the method (Fig. 8). Structures with the high aspect ratio (10), located too close to each
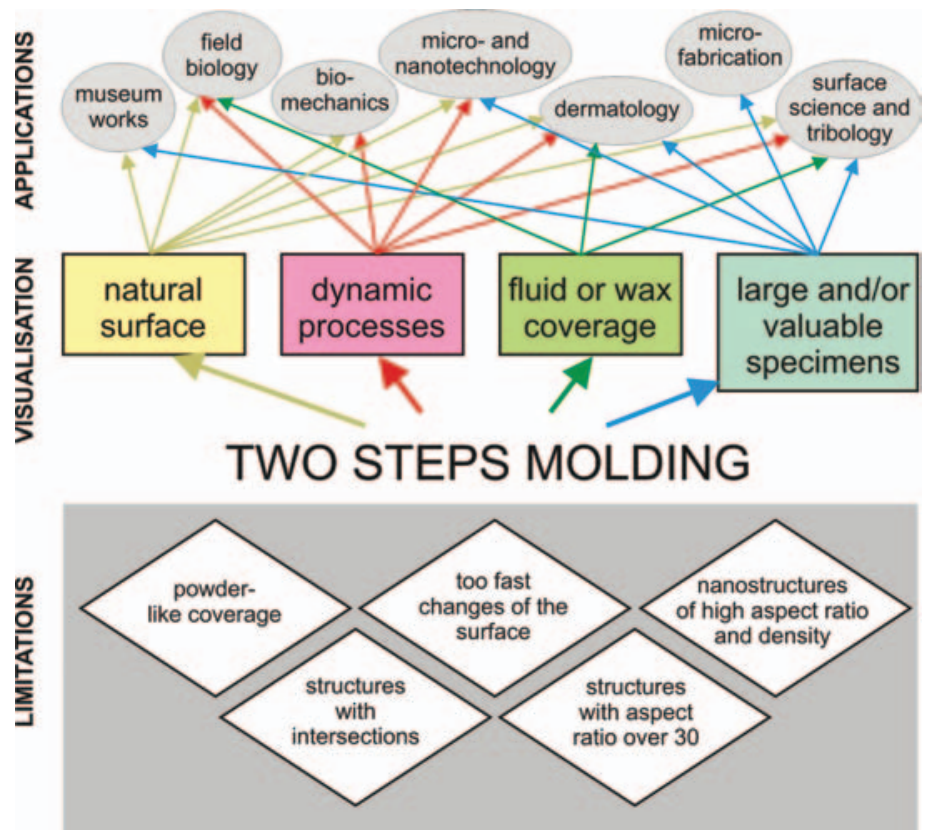

Fig. 8. Diagram showing visualisation purposes for different fields of application of the two steps molding method for SEM. In the lower part of the diagram, limitations of the method are listed.

other (gaps under $100 \mathrm{~nm}$ ), were difficult to replicate, because of the limited wettability of both the fluid PVS and Epoxy (Fig. 2 C, D). Surfaces covered with solid particles insoluble with organic solvents cannot be properly replicated. Visualisation of droplets having low viscosity is possible in principle, but the precise replication of the contact angle of the droplets is not free of artefacts, because the interactions between both molding contacting fluids are unknown. Surfaces changing their profile faster than polymerisation time of the PVS cannot be properly replicated.

\section{Literature}

Ensikat, H.J., and Barthlott,W. Liquid substitution: a versatile procedure for SEM specimen preparation of biological materials without drying and coating. $J$. Microsc. 172: 195-203, 1993.

Green P.B., and Linstead, P. A procedure for SEM of complex shoot structures applied to the inflorescence of snapdragon (Antirrhinum). Protoplasma. 158: 33-38, 1990.

Gorb, S.N. Ultrastructure of the thoracic dorso-medial field (TDM) in the elytra-tobody arresting mechanism in tenebrionid beetles (Coleoptera: Tenebrionidae). J. Morphol. 240: 101-113, 1999a.

Gorb, S.N. Evolution of the dragonfly head-arresting system. Proc. Roy. Soc. London B 266: 525-535, 1999b.

Gorb, S.N. Attachment devices of insect cuticle. Dordrecht, Boston, London:Kluwer Academic Publishers. 1-305, 2001.

Gorb, E., and Gorb, S. Contact separation force of the fruit burrs in four plant species adapted to dispersal by mechanical interlocking. Plant Physiol. Biochem. 40: 373-381, 2002.

Gorb, S.N., Jiao, Y., and Scherge, M. Ultrastructural architecture and mechanical properties of attachment pads in Tettigonia viridissima (Orthoptera Tettigoniidae). J. Comp. Physiol. A 186: 821-831, 2000.

Koch, K., Dommisse, A., Barthlott, W., and Gorb, S.N. The use of plant waxes as templates for micro- and nanopatterning of surfaces. Bioinspiration and Biomimetics, 2007, in press.

Spurr, A.R. A low-viscosity epoxy resin embedding medium for electron microscopy. J. Ultrastr. Res. 26: 31-43, 1969.

Scherge, M., and Gorb, S.N. Biological micro- and nanotribology. Berlin: Springer, 2001.

Schwarz, H., and Gorb S.N. Method of platinum-carbon coating of ultrathin sections for transmission and scanning electron microscopy: An application for study of biological composites. Microsc. Res. Techn. 62: 218-224, 2003. 


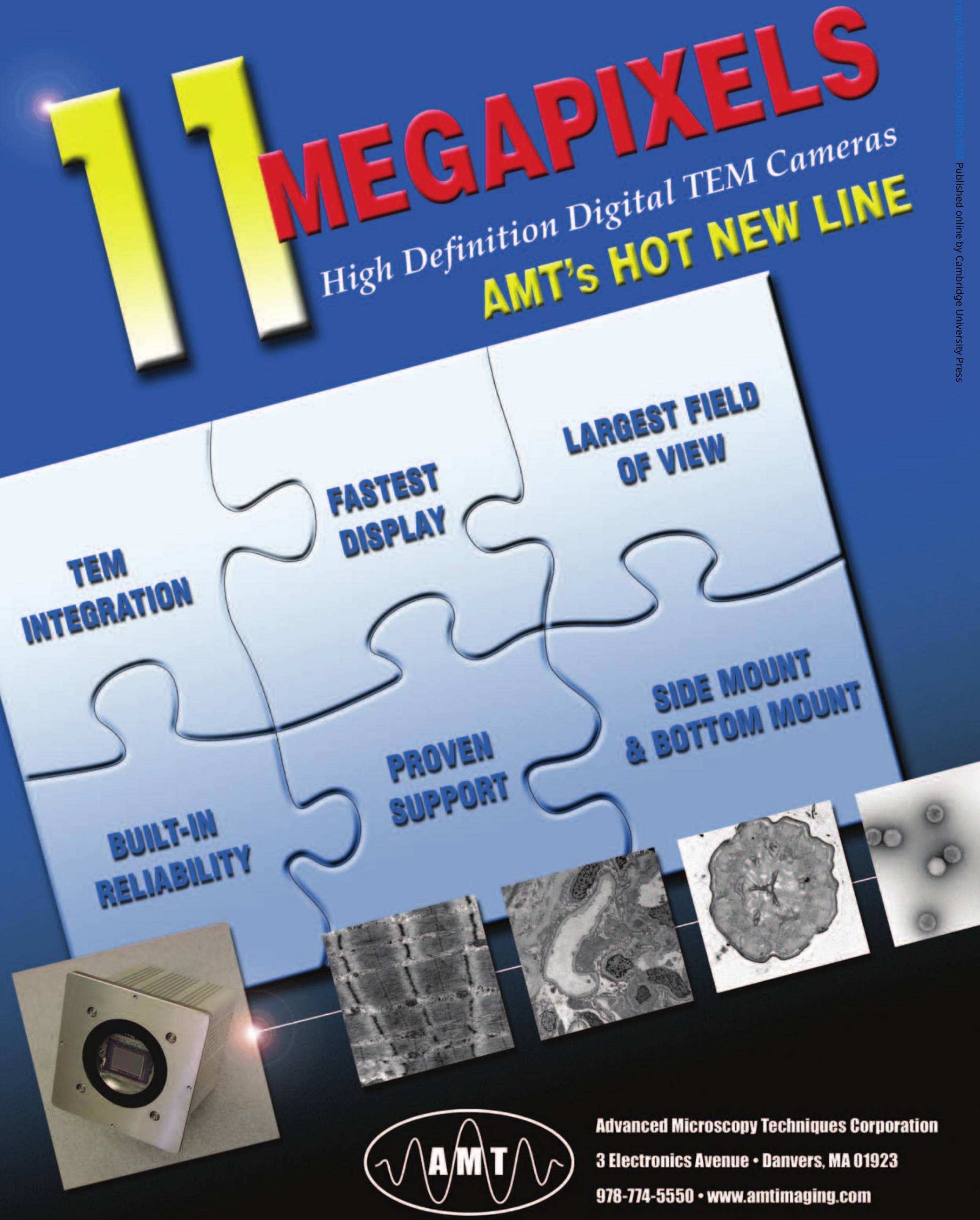

